\title{
The Application of Jade Prosthesis and Allograft in Proximal Tibia of Neoplastic Bone Defect: A Case Report
}

\author{
Jiazuo Shen, Jianfei Yao, Dasheng Lin, Zhenqi Ding, Kejian Lian*, Bin Lin \\ Department of Orthopaedic Surgery, the Affiliated Dongnan Hospital of Xiamen University, Orthopaedic Trauma Center of the 175th \\ Hospital of PLA, Zhangzhou, Fujian, PR China \\ *Corresponding author: 24520091152983@stu.xmu.edu.cn
}

Received April 24, 2013; Revised June 01, 2013; Accepted June 02, 2013

\begin{abstract}
The upper-middle tibia is the multiple growth section of benign and malignant neoplasm, which is easy to result in bone defect after tumorectomy and it is a serious problem in orthopaedics. We reported a case about a huge benign or potential malignant tumor( giant cell tumor of bone) in upper-middle tibia and contrasted the advantage between the jade prosthesis and the allograft. Initially, the tumor had been curetted and then the uppermiddle tibia had been given iliac bone graft, but the tumor recurred one year later. Then orthopedists gave the tumor excision and jade prosthesis replacement by the fixation of intramedullary screw. However, twenty one years later, the intramedullary screw fatigue fractured, orthopedists advised the patient be unloaded the jade prosthesis and be given the operative knee prosthesis surface replacement before allogeneic tibial condyle replacement. While 6 months later, the allogeneic bone in vivo had immunologic rejection and the knee was swelling, pain and no function. Finally, orthopedists gave the knee arthrodesis. Hence, we discovered that jade prosthes is had more advantages than allograft in proximal tibia of neoplastic bone defect and jade prosthesis was secure, durable, long period length, low immunological rejection and should be popularized in clin ic.
\end{abstract}

Keywords: jade prosthesis, knee surface arthroplasty, allogeneic bone, giant cell tumor of bone, arthrodesis

\section{Introduction}

The upper-middle tibia is the multiple growth section of benign and malignant neoplasm, which is one of the prime reasons about pain, swelling and dysfunction for the kneejoint and the upper-middle tibia. Giant cell tumor of bone(GCT) is a primary benign and potential malignant bone neoplasm, which mainly invade in long bones, especially invade in lower femur and upper-middle tibia. Surgical extremity amputation was the main therapy century ago. For almost two decades extremity amputation has not been the only viable option for patients with bone tumor in the region of the hip and knee. Remarkable advances in implant technology, surgical reconstructive technique and adoption of new chemotherapy protocols provides a new option for surgeons who diagnose and treat bone tumors [1]. Jade prosthesis and allogeneic bone graft had become accepted alternative in limb salvage surgery of the extremities [2]. They allow restoration of function, improve the control of malignant disease and subjective patient satisfaction.

\section{Case Presentation}

22 years ago, a 31-year-old female patient visited our orthopaedic center with complaints of increasing joint soreness and limitation of motion of the right knee. On auxiliary examination, we discovered GCT in the right upper-middle tibia by X-ray examination and GCT may invaded tibial plateau so orthopedists in our center advised the patient hospitalization to have an operation. Then orthopedists curetted the tumor and cauterized the tumor boundary by phenol and grafted the iliac bone. But one year later, GCT recurred again, orthopedists discussed to amputate the upper-middle tibia to control the tumor diffuse and improve patients life quality. A substantial amount of healthy bone may need to be resected to provide a safe margin, leaving a remnant bone segment enough to secure fixation of prosthesis [3].Then the patient had been given arthroplasty replacement by jade prosthesis and fixed with the jade prosthesis by intramedullary screw and bone cement transplantation. Postoperatively, the patient had a satisfactory subjective sensation, recovered and discharged from hospital. GCT had not recurred and the function of the knee recovered to nomal level in the next 21 years.

Half a year ago, the patient visited our orthopaedic center again with complaints of increasing joint soreness, obviously swell and limitation of motion in the right operative knee. On X-ray examination, the jade prosthesis loosed around and the intramedullary screw fatigue fractured in the end (Figure 1), orthopedist proposalled the patient to be in hospital and operation again. This time jade prosthesis together with intramedullary screw was dislodged away and an allogenetic tibial condyle, which 
had sterilization and disinfection and freezing to $-80^{\circ}$ and was similar with condyles of tibia of the patient both the length and the breadth, was fixed with an inner tibial anatomical locking plate and then had knee surface replace ment operation(Figure 2). When the jade prosthesis and the intramedullary screw were dislodged, we saw some soft tissues surrounding the jade prosthes is and sent these tis sues for pathological examination(Figure 3-I).
However, the knee persistently swell and had no function and the wound had disunion and had inflammatory reaction. After 6 times debridement operation, the allogenetic bone had immunologic rejection and bone resorption 6 months postoperatively, orthopedists in our center gave the knee arthrodesis finally (Figure 4) and sent the tissues for pathological examination(Figure $3-\mathrm{J}$ ).
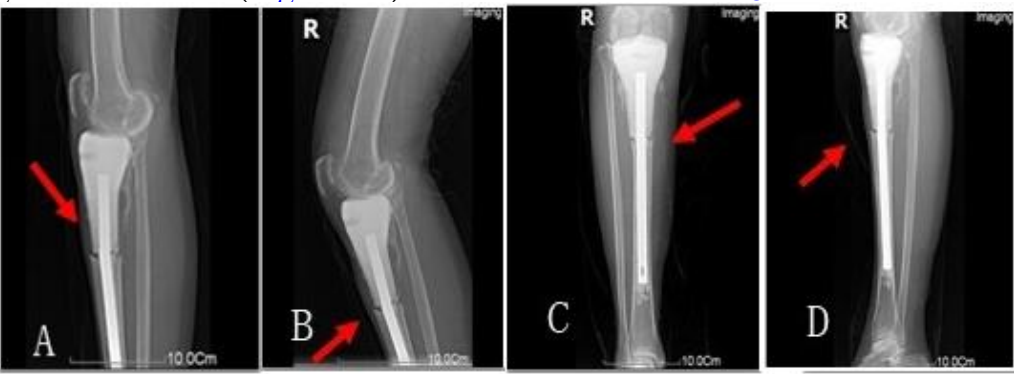

Figure 1. A、B、C、D all shows the X-ray photographs of jade prosthesis replacement after 21 years later that the intramedullary screw fatigue fractured

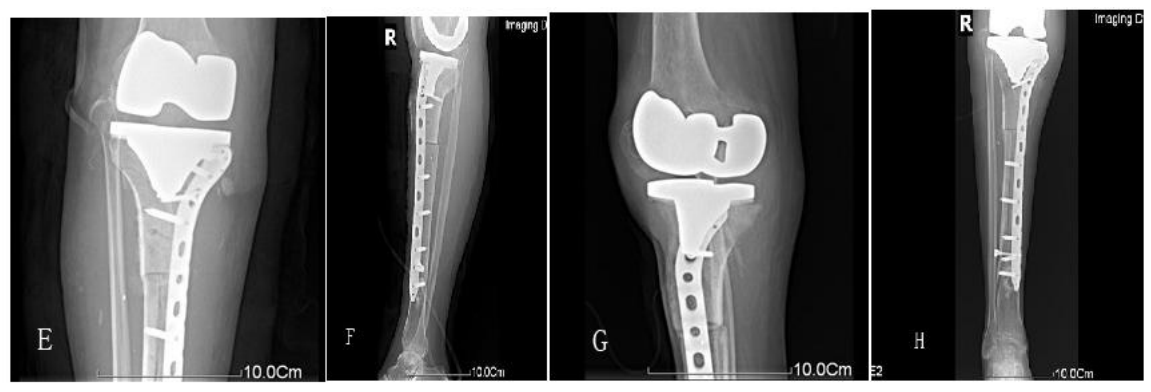

Figure 2. E and $\mathrm{F}$ show allogenetic tibial condyle graft and tibial anatomical locking plate fixation and knee surface replacement on the next day postoperatively; $\mathrm{G}$ and $\mathrm{H}$ show 6 months later postoperat ively

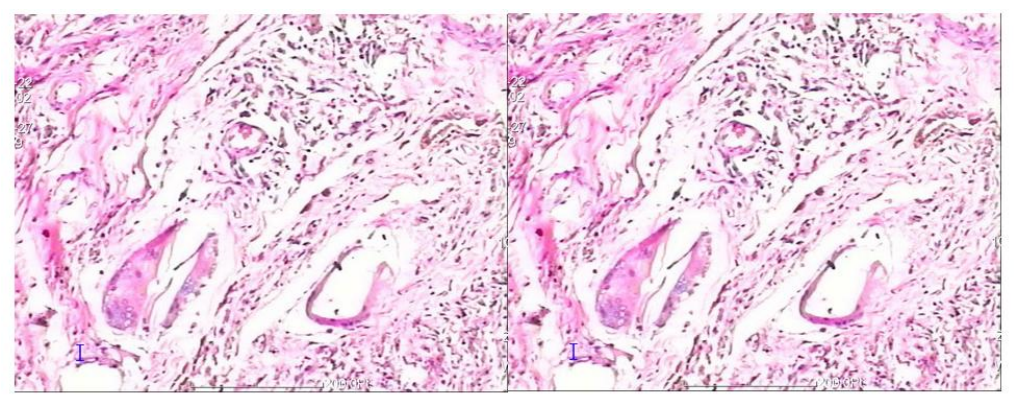

Figure 3. I shows pathological examination after operation at allogenetic tibial condyle graft and tibial anatomical locking plate fixation and knee surface replacement. Histopathological picture was $H E \times 100$ and shows plentiful of inflammatory cells, hemosiderin cells, gaint cells, foreign body giant cell reaction, metamorphic bone tissue, which may diagnose pigment um vilonodular synovitis of the knee that infiltrates the soft tissues around articular cavity and adjacent bone tissues

$\mathrm{J}$ shows pathological examination after operation at knee arthrodesis operation. Histopathological picture was $H E \times 100$ and shows loose connective tissue edema and plentiful of inflammatory cells, hemosiderin cells, multifocal flake necrosis cells, interstitial vascular proliferation, metamorphic bone tissue and calcification which may diagnose chronic granuloma
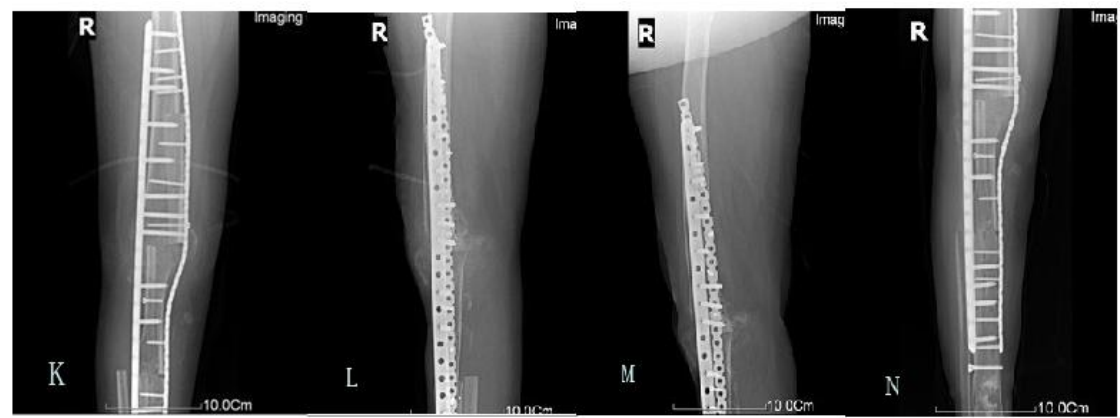

Figure 4. $\mathrm{K}$ and $\mathrm{L}$ show knee arthrodesis on the next day postoperatively; $\mathrm{M}$ and $\mathrm{N}$ show 6 months later postoperatively 


\section{Discussion}

Lower-middle femur and upper-middle tibia are the main location of bone tumors. According to the benign and malignant of the tumors and biological behavior characteristics of the tumors, specialists have different therapeutic advices, which mainly contain tumor margin inactivation before the tumors had been curetted and function reconstruction before tumors had been excised. But by comparison of several studies on the results of inactivation treatment for tumors, a local recurrence of $27.0 \%$ after curettage only, $25.0 \%$ after phenol application, $7.9 \%$ after cryosurgery, while $0 \%$ after wide enbloc excision $[4,5,6]$.

On this case report it is GCT which is a common primary bone tumor in clin ic, the biological behavior have some invasiveness characteristics. Though the tumor had been curetted and inactivated the margin by phenol during the operation, it can not reduce the recurrence rate in clinic [5], which may be $25 \%$ after phenol application while $0 \%$ after wide enbloc excision [6]. However, twenty one years ago, it restricted by orthopedic instrument and was lack of basic theory and technical level, orthopedists endeavored to resect the tumor, reserve the limb and attempt the jade prosthes is to replace amputation so as to reserve the limb function. During twenty one years, jade prosthesis replacement attempt had been certified successfully and provided precious experience for prosthesis replacement in orthopedics.

Jade prosthesis is a natural jade and manufactured to various kinds of shapes to coincide with human bones. It can resist acid base corrosion, has no apparent immunologic rejection and mainly consists of hydrosilicate and its chemical composition contains a small quantity of sodium, calcium, magnesium, alu minum and other trace elements. After sterilization and disinfection the jade is used as a medical prosthesis to replace the tumor location. For twenty one years clinical observation jade prosthesis has been certified to have a predominant biocompatibility and biomechanics.

On this case, jade prosthes is has existed as usual in vivo for twenty one years and the knee function recovered well but the intramedullary screw was fatigue fractured in the end. Orthopedists had better unload the jade prosthesis together with intramedullary and give the operative knee prosthesis surface replacement before allogeneic tibial condyle replacement. However, allogeneic tibial condyle in vivo had immunological rejection and the allogeneic tibial condyle absorbed to sink. Finally, the knee had no function and swell, orthopedists had better give the knee arthrodesis.
Through this case report, we discovered that jade prosthesis had more advantages than allogeneic bone in proximal tibia of neoplastic bone defect in clinic. Jade prosthesis was secure, durable, long period length, low immunological rejection, solve the problem of neoplastic bone defect in clinic and should be popularized in clinic. However, there are still some deficiencies: the case is less, lack of large sample research and need more clinical research to confirm the security and reliability of the jade prosthesis, which is the next research content of our orthopedic center.

\section{Conclusion}

Jade prosthesis replacement might be an elective method for bone defect to reconstruct knee function and it was somewhat better than allogeneic bone graft, which might exist immunological rejection to some extent.

\section{Authors' Contributions}

SHEN Jia-zuo and YAO Jian-fei contributed equally to the work. SHEN Jia-zuo wrote the article. DING Zhen-qi carried out the operation. All authors read and approved the final manuscript.

\section{Conflict of Interest}

The authors have declared that no conflict of interest exists.

\section{References}

[1] Barjaktarovic R, Popovic Z, Radoicie D. Megaendoprosthesis in the treatment of bone tumors in the knee and hip region. Vojnosanit Pregl. 2011. 68(1): 62-7

[2] Tan PK, Tan MH. Functional outcome study of megaendoprosthetic reconstruction in limbs with bonetumour surgery. Ann Acad Med Singapore. 2009. 38(3): 192-6.

[3] Jeys LM, Grimer RJ, Carter SR, Tillman RM, Abudu A. Post operative infection and increased survival in osteosarcoma patients: arethey associated. Ann Surg Oncol. 2007. 14(10): 2887-95.

[4] Boons HW, Keijser LC, Schreuder HW, Pruszczynski M, Lemmens JA, Veth RP. Oncologic and functional results after treatment of giant cell tumors of bone. Arch Orthop Trauma Surg. 2002. 122(1): 17-23.

[5] Trieb K, Bitzan P, Lang S, Dominkus M, Kotz R. Recurrence of curetted and bone-grafted giant-cell tumours with and withoutadjuvant phenol therapy. Eur J Surg Oncol. 2001. 27(2): 200-2.

[6] Veth R, Schreuder B, van BH, Pruszczynski M, de Rooy J. Cryosurgery in aggressive, benign, and low-grade malignant bone tumours. Lancet Oncol. 2005. 6(1): 25-34. 